\title{
Review: cognitive therapy may be no more effective than other bona fide psychological therapies for depression
}

Wampold B, Minami T, Baskin T, Tierney S. A meta-(re)analysis of the effects of cognitive therapy versus 'other therapies' for depression. Journal of Affective Disorders 2002 April; 68: 159-165.

\section{QUESTION: Is cognitive therapy more effective than other psychological treatments for depression?}

\section{Design}

Reanalysis of meta-analysis conducted in 1998.

\section{Data sources}

The authors do not describe the data sources used in the original meta-analysis (the search strategy is outlined in a previous article).

\section{Study selection}

Eligible studies were randomised trials comparing cognitive therapy with another psychological treatment for people with a major depressive or dysthymic disorder. People with psychotic or bipolar disorders were excluded. 22 studies were included in the original meta-analysis, 10 of which used a 'bona fide' comparison group. Treatments were defined as 'bona fide' if the therapist was trained to provide therapy, held at least a masters degree or was enrolled in a relevant graduate programme; face to face meetings were used to develop a relationship; treatment was individualised for the participant rather than involving a rigid standard protocol, and therapy included psychologically valid components.

\section{Data extraction}

Data were extracted on population, sample size, treatment characteristics and effect size. Larger studies were more heavily weighted when calculating aggregate effect sizes. Depression was assessed using the Beck Depression Inventory in all studies.

\section{Main results}

Cognitive therapy and 'other therapies' were equally effective for reducing depression scores. The aggregate effect size of cognitive therapy was 0.49 over non bona fide therapies (95\% CI 0.28 to 0.69 ); 0.16 over bona fide therapies (95\% CI - 0.01 to 0.32 ), and 0.03 over bona fide therapies once a statistical outlier was removed (95\% CI -0.15 to 0.2$)$.

\section{Conclusions}

Previous meta-analyses suggested that cognitive therapy is more effective than other therapies for depression (excluding behaviour therapy). After accounting for 'control' treatments, this reanalysis found no evidence that cognitive therapy was more effective than other psychological therapies. The authors conclude that bona fide psychological treatments for depression are equally effective.

\section{COMMENTARY}

There is controversy over the relative efficacy of psychological therapies. ${ }^{1}$ Some emphasize common features of psychotherapeutic techniques, particularly the quality of the therapeutic relationship,,$^{2-3}$ while others explore the superiority of one technique over another. Cognitive behaviour therapy and interpersonal psychotherapy have been evaluated extensively and are often regarded as more 'evidencebased' than older therapies.

A range of therapies is probably needed to ensure the best match with an individual patient. Clarifying the 'added value' of any therapy over another for a particular individual and their therapist remains a research challenge. This paper contributes to the debate by offering a valid critique of Gloaguen et al's influential earlier meta-analysis. In contrast to the original findings, cognitive therapy was not superior to 'other therapies' once control treatments or apparently inadequate therapies were excluded. Although this reanalysis is interesting, it has several limitations:

i) The number of participants receiving other therapies was not large ( $\mathrm{n}=265$ in 9 studies in the final analysis). ${ }^{4}$

ii) There was a small advantage to cognitive therapy until one of the original 10 studies was excluded as a statistical outlier.

iii) The most recent study was published in 1994 .

iv) 'Other approaches' included both individual and group treatments, ranging from psychodynamic models to highly structured interpersonal psychotherapy.

v) As well as using the Beck Depression Inventory, other measures of psychosocial functioning and longer-term follow up may have revealed important differences.

The findings suggest that a range of treatments have similar therapeutic benefit. If recent breakdowns of the efficacy of 'cognitive' and 'behavioural' components of cognitive behavioural therapies are anything to go by, it will be difficult to identify the key elements of effective treatments. We cannot draw conclusions from this review about the relative merit of other therapies due to heterogeneity and the small number of studies. Unfortunately this does not assist us in selecting therapy for individual patients.

ProfessorPete M Ellis, BM BCh PhD FRANZCP Department of Psychological Medicine Wellington School of Medicine, New Zealand

1 Holmes J. All you need is cognitive behaviour therapy? BMJ 2002; 324: 288-90

Stiles WB, Agnew-Davies R, Hardy GE et al. Relations of the alliance with psychotherapy outcome: Findings in the second Sheffield psychotherapy project. J Consult Clin Psychology 1998; 66: 791-802

3 Wiser S, Goldfried MR. Therapist interventions and client emotional experiencing in expert psychodynamicemotional experiencing in expert psychodynamicnterpersonal and cognitive-behavioural therapies. Consult Clin Psychology 1998; 66: 634-40.

Gloaguen V, Cottraux J, Cucherat $\mathrm{M}$ et al. A meta-analysis of 作 tive Dis 1998; 49: 59-72.
Sources of funding: Not specified

For correspondence: $B$ Wampold,

Department of Counseling Psychology, University of Wisconsin, Madison wampold@ education.wisc.edu 